\title{
Carbon-based conductive materials accelerated methane production in anaerobic digestion of waste fat, oil and grease
}

\author{
Xia $\mathrm{He}^{\mathrm{a}}$, Zhenyu Guo ${ }^{\mathrm{a}}$, Jian Lu ${ }^{\mathrm{b}, \mathrm{c}^{*}}$, Ping Zhang ${ }^{\mathrm{a}}$ \\ ${ }^{a}$ College of Environmental Science and Engineering, Guilin University of Technology, Guangxi 541006, China \\ ${ }^{\mathrm{b}}$ CAS Key Laboratory of Coastal Environmental Processes and Ecological Remediation, Yantai Institute of Coastal Zone Research (YIC), Chinese Academy of Sciences \\ (CAS), Shandong Key Laboratory of Coastal Environmental Processes, YICCAS, Yantai, Shandong 264003, China \\ ${ }^{\mathrm{c}}$ Center for Ocean Mega-Science, Chinese Academy of Sciences, 7 Nanhai Road, Qingdao 266071, China
}

\section{H I G H L I G H T S}

- Nano-graphite, activated carbon, and carbon cloth were used to enhance co-digestion.

- Application of carbon materials led to $13-22 \%$ incremental methane production.

- DIET-mediated syntrophic acetate oxidation (SAO) enabling faster acetate conversion.

- Functional microbes involved in DIET-mediated SAO were dependent on carbon materials.

\section{A R T I C L E I N F O}

\section{Keywords:}

Anaerobic digestion

Conductive materials

Grease

Sludge

Direct interspecies electron transfer

\begin{abstract}
A B S T R A C T
Little is known about the effect of carbon-based conductive material (CM) addition on the anaerobic co-digestion of fat, oil and grease (FOG) and waste activated sludge (WAS). In this study, three types of carbon-based CMs (nano-graphite (NG), granular activated carbon (GAC), and carbon cloth (CC)) and nine dosages were evaluated for their influences on co-digestion performance. The best dosage was achieved at $0.2 \mathrm{~g} / \mathrm{L} \mathrm{NG}, 10 \mathrm{~g} / \mathrm{L} \mathrm{GAC}$, and 1 $\mathrm{cm} \times 5 \mathrm{~cm}$ CC with $13-22 \%$ incremental methane production, $25-55 \%$ increased VS removal and $28-32 \%$ enhanced COD conversion efficiency compared to the control. The highest total amount of bacteria/archaea was found in CC $(1 \mathrm{~cm} \times 5 \mathrm{~cm})$, followed by GAC at $10 \mathrm{~g} / \mathrm{L}$ and NG at $0.2 \mathrm{~g} / \mathrm{L}$, which were all higher than those of the control. Microbial community analysis revealed that direct interspecies electron transfer (DIET)-mediated syntrophic acetate oxidation (SAO) enabling faster acetate conversion might be responsible for the enhancement of methane production.
\end{abstract}

\section{Introduction}

Increasing attention has been paid to the harmful organic waste disposal and clean bioenergy recovery in recent years (Lu et al., 2021). The anaerobic digestion has been proved to be a promising approach for organic waste disposal and bioenergy recovery (Salama et al., 2019). Fat, oil and grease (FOG) are primarily discharged from food service establishments at a rate of approximately $7.1 \mathrm{~L} /$ person/year (He et al., 2017; Long et al., 2012), which possess high biomethane yield potential at $1014 \mathrm{~mL} / \mathrm{g}$ volatile solid (VS) compared to proteins at $740 \mathrm{~mL} / \mathrm{g}$ VS and carbohydrates at $370 \mathrm{~mL} / \mathrm{g}$ VS (Harris and Mccabe, 2015). Anaerobic co-digestion of FOG and waste activated sludge (WAS) from wastewater treatment plants (WWTPs) has been recognized as an attractive application for FOG disposal because it provides high $\mathrm{C} / \mathrm{N}$ ratio of co-substrates, dilutes the inhibitory or toxic compounds, improves the energy yield, and decreases the environmental of landfill as collected FOG are usually landfilled (Mata-alvarez et al., 2014; Salama et al., 2019).

Long chain fatty acids (LCFAs), product of FOG hydrolysis, are the vital intermediates during anaerobic digestion of FOG, which can adsorb onto biomass resulting in sludge flotation, biomass washout, and the inhibitory effects on key microbes (e.g., methanogens) (Silva et al., 2016; Sousa et al., 2009). Accumulation of LCFAs is considered to be the major cause for the reduced methane production rate in anaerobic

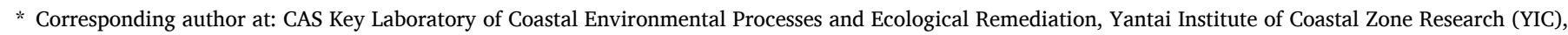
Chinese Academy of Sciences (CAS), Shandong Key Laboratory of Coastal Environmental Processes, YICCAS, Yantai, Shandong 264003, China.

E-mail address: jlu@yic.ac.cn (J. Lu).
} 
digestion of FOG, which prolongs the lag phase and decreases the stability of the anaerobic digesters (Palatsi et al., 2009; Salama et al., 2019; Wu et al., 2018). In spite of LCFAs, ammonia derived from WAS may be another major inhibitor of methanogenesis (especially for acetoclastic methanogens) (Rajagopal et al., 2013), which needs to be addressed to avoid inefficient operation. Thus, methods to overcome the inhibition would have a significant advantage for accelerated methane production and more stable operation of anaerobic co-digestion of FOG and WAS.

The efficiency of anaerobic digestion is primarily dependent on the syntrophic interactions between bacteria and methanogens (Martins et al., 2018). Recently, many studies have suggested the addition of carbon-based conductive materials (CMs) can promote direct interspecies electron transfer (DIET), which has been proposed as a faster and potentially more energy-conserving electron transfer strategy compared to the interspecies hydrogen/formate electron transfer (Cruz Viggi et al., 2014; Lovley, 2017), resulting in shorten lag phase and accelerated methane production rate (Wu et al., 2020; Zhang et al., 2018). Meanwhile, the addition of CMs can also promote the electron transfer, which subsequently enhances the removal of persistent contaminants such as tetrabromobisphenol A (Zhang et al., 2020a). Nevertheless, other results suggested that the effect of CMs goes beyond the stimulation of DIET (Martins et al., 2018), and specific mechanism of the CMs enhancement on anaerobic digestion is not yet clear, especially for the aspects of functional electron carriers, microbial interaction, and the impact of organic substrates on DIET. To better understand this, microbial community needs to be investigated to identify the important microbial players and get insights into the interspecies electron transfer interactions. Besides, only few studies have reported the effect of CMs on the anaerobic digestion of lipid-waste (e.g., waste oil) (Chowdhury et al., 2019; Lü et al., 2019; Zhang et al., 2020b). Little is known about the effect of carbon-based CM addition on the anaerobic co-digestion of FOG and WAS.

The main purpose of this study was to investigate the effect of carbon-based CMs on the methane production and complex microbial communities during anaerobic co-digestion of FOG and WAS, including determining the influence of carbon-based CMs on co-digestion performance, investigating the physical and chemical properties of CMs and microbial colonization, and elucidating the microbial community structure and the possible microbial interactions using 16S rRNA gene sequencing of Bacteria and Archaea communities. The results of the research are expected to provide insights into the mechanism of carbonbased CMs enhancement on anaerobic co-digestion of FOG and WAS as well as instruction for parameter optimization of CM-amended oily waste treatment.

\section{Materials and methods}

\subsection{Preparation of inoculum, substrate and conductive materials}

Sludge obtained from the upflow anaerobic sludge blanket reactor of a brewery wastewater treatment (Liquan Beer Co. Ltd, Guilin, China) was used as inoculum. The WAS used for this study was taken from Qilidian WWTP (Guilin, China) using a conventional activated sludge process at a design flow of $100,000 \mathrm{~m}^{3}$ /day with a hydraulic retention time of 0.5 day. All samples were quickly transferred to the laboratory after collection and stored at $4{ }^{\circ} \mathrm{C}$ for no more than 3 days before utilization. Blending oil (Jinlongyu Co. Ltd, Shanghai, China), a mixture of 8 types of oil including peanut oil, soybean oil, canola oil, sunflower seed oil, rice bran oil, corn oil, sesame oil and flaxseed oil, was used as a model compound for FOG. The characteristics of the inoculum and substrates used in this study were determined (see supplementary material). Three kinds of carbon-based CMs were used in this study, including nanoparticles of graphite (NG) (XF011, XFNANO Materials Tech Co. Ltd, Nanjing, China), granular activated carbon (GAC) (Aladdin, Shanghai, China), and carbon cloth (CC) (Lishuo Composite Material Tech Co. Ltd, Shanghai, China).

\subsection{Experimental design and setup}

Glass serum bottles of $200 \mathrm{~mL}$ with a working volume of $150 \mathrm{~mL}$ were used as reactors and all treatments were performed in triplicate. Three types of CMs with different dosages were separately added to the reactors to evaluate their effects on the methane production during anaerobic co-digestion of FOG and WAS (Table 1). Inoculum without substrate and CM addition was used as blank, and inoculum with the addition of FOG and WAS but without CM was used as control. To maintain anaerobic conditions, the bottles were flushed with $\mathrm{N}_{2}$ gas for $5 \mathrm{~min}$ prior to being sealed. All bottles were incubated in a temperaturecontrolled shaker (ZD-85, Jinyi instrument Co. Ltd, Changzhou, China) at $37{ }^{\circ} \mathrm{C}$ with a mixing speed of $150 \mathrm{rpm}$. The volume of biogas in each bottle was measured periodically by releasing the pressure in the bottle using a $10 \mathrm{~mL}$ gas-tight plastic syringe, and the composition of biogas was immediately analyzed. Liquid samples within all bottles were collected at the end of incubation for measurements of $\mathrm{pH}$, ammonia, chemical oxygen demand (COD), total solids (TS), volatile solids (VS) and volatile fatty acids (VFAs). Samples of the CMs in all bottles were also collected for further analysis using scanning electron microscopy (SEM). Digestion tests were run until the daily methane production of each bottle less than $1 \%$ of the cumulative methane production.

\subsection{Analytical methods}

\subsubsection{Physiochemical analysis}

The TS, VS, ammonia, and alkalinity were analyzed according to Standard Methods for the Examination of Water and Wastewater (APHA, 1999). COD was analyzed using test kits (Ultra High Range, HACH, Loveland, CO, USA) with a HACH DRB200 COD Reactor and a HACH DR3900 spectrophotometer. Biogas production was recorded and normalized to STP conditions based on the local climatological data. The composition of biogas was analyzed using a gas chromatograph (GC126N, INESA, Shanghai, China) equipped with a thermal conductivity detector and a $2 \mathrm{~m} * 3 \mathrm{~mm}$ TDX-01 column (INESA, Shanghai, China). The volatile fatty acids (VFAs) were determined using the direct injection method as previously described ( $M u$ et al., 2018). The composition of VFAs was characterized by GC-FID (6890B, Agilent Technologies, Palo Alto, CA, USA) using helium as carrier gas and a capillary column (DB-FFAP, $30 \mathrm{~m} \times 0.25 \mathrm{~mm} \times 0.25 \mathrm{um}$, Agilent Technologies, Palo Alto, CA, USA). To visualize cell attachment to CMs,

Table 1

Composition of inoculum, substrate and materials in the digestion experiments.

\begin{tabular}{lllllll}
\hline & Materials & $\begin{array}{l}\text { Inoculum } \\
(\mathrm{mL})\end{array}$ & $\begin{array}{l}\text { WAS } \\
(\mathrm{mL})\end{array}$ & $\begin{array}{l}\text { FOG } \\
(\mathrm{mL})\end{array}$ & $\begin{array}{l}\text { S/I } \\
\text { ratio }\end{array}$ & $\begin{array}{l}\text { WAS } \\
\text { proportion in } \\
\text { substrate(VS } / \\
\text { VS) }\end{array}$ \\
\hline Blank & & 15 & 0 & 0 & - & - \\
Control & & 15 & 5 & 0.24 & 0.5 & 0.22 \\
NG1 & $\begin{array}{l}0.2 \mathrm{~g} / \mathrm{L} \\
\mathrm{NG}\end{array}$ & 15 & 5 & 0.24 & 0.5 & 0.22 \\
$\mathrm{NG} 2$ & $\begin{array}{l}0.5 \mathrm{~g} / \mathrm{L} \\
\mathrm{NG}\end{array}$ & 15 & 5 & 0.24 & 0.5 & 0.22 \\
$\mathrm{NG} 3$ & $\begin{array}{l}1.0 \mathrm{~g} / \mathrm{L} \\
\mathrm{NG}\end{array}$ & 15 & 5 & 0.24 & 0.5 & 0.22 \\
$\mathrm{GAC} 1$ & $\begin{array}{l}5.0 \mathrm{~g} / \mathrm{L} \\
\mathrm{GAC}\end{array}$ & 15 & 5 & 0.24 & 0.5 & 0.22 \\
$\mathrm{GAC} 2$ & $\begin{array}{l}10.0 \mathrm{~g} / \mathrm{L} \\
\mathrm{GAC}\end{array}$ & 15 & 5 & 0.24 & 0.5 & 0.22 \\
$\mathrm{GAC} 3$ & $\begin{array}{l}15.0 / \mathrm{L} \\
\mathrm{GAC}\end{array}$ & 15 & 5 & 0.24 & 0.5 & 0.22 \\
$\mathrm{CC} 1$ & $\begin{array}{l}1 \mathrm{~cm} * 1 \mathrm{~cm} \\
\mathrm{CC}\end{array}$ & 15 & 5 & 0.24 & 0.5 & 0.22 \\
$\mathrm{CC} 2$ & $\begin{array}{l}1 \mathrm{~cm} * 3 \\
\mathrm{~cm} \mathrm{CC}\end{array}$ & 15 & 5 & 0.24 & 0.5 & 0.22 \\
$\mathrm{CC} 3$ & $\begin{array}{l}1 \mathrm{~cm} * 5 \\
\mathrm{~cm} \mathrm{CC}\end{array}$ & 15 & 5 & 0.24 & 0.5 & 0.22 \\
\hline
\end{tabular}

$\mathrm{NG}=$ Nano-graphite, GAC $=$ granular active carbon, $\mathrm{CC}=$ carbon cloth. 
samples were prepared according to previously described method (Chen et al., 2014), and the scanning electron micrographs were taken using Field Emission Scanning Electron Microscope (ZEISS Sigma, Germany).

\subsubsection{Kinetic analysis}

A modified Gompertz model (Eq. (1)) (Li et al., 2011) was adopted to elucidate the kinetics of methane production during the anaerobic codigestion experiment, where $B_{(t)}$ is the specific methane yield at a given time (mL/gVS added); $B_{0}$ is the maximum methane potential (mL/ $\mathrm{gVS}_{\text {added }}$ ); $t$ is the digestion time since the start of the tests (d); $R_{m}$ is the maximum daily methane production rate $\left(\mathrm{mL} / \mathrm{gVS}_{\text {added. }} \mathrm{d}\right) ; \lambda$ is the lagphase (d); $e$ is 2.718 .

$B_{(t)}=B_{0} \exp \left\{-\exp \left[\frac{R_{m} e}{B_{0}}(\lambda-t)+1\right]\right\}, t \geq 0$

\subsubsection{Microbial analysis}

Mixed liquor samples from inoculum and final solution of Control, NG1, GAC2, and CC3 (CC3-S), as well as the attached biomass along with the carbon cloth (CC3-A) were collected for microbial analysis. Briefly, DNA was extracted using CTAB method (Wirth et al., 2012). Ion torrent sequencing was conducted for the samples at the Sequencing Services Facility at the Novogene Co., Ltd (Beijing, China). Amplicon libraries were constructed using the primer set $341 \mathrm{~F} / 806 \mathrm{R}$ for Bacteria (Sundberg et al., 2013) and the primer set 519F/915R for Archaea (Fan and Xing, 2016). Operational taxonomic units (OTUs) clustering and taxonomy identification were performed as previously described (Hao et al., 2020). The compositional differences between microbiomes were analyzed by principal coordinate analysis (PCoA) using R Phyloseq package based on Bray-Curtis distance (Navas-Molina et al., 2013). Sequences were submitted to the NCBI Sequence Read Archive as BioProject PRJNA675673. Furthermore, bacterial and archaeal quantity in the DNA samples were evaluated by quantitative polymerase chain reaction (qPCR) analysis as previously described (Lü et al., 2019).
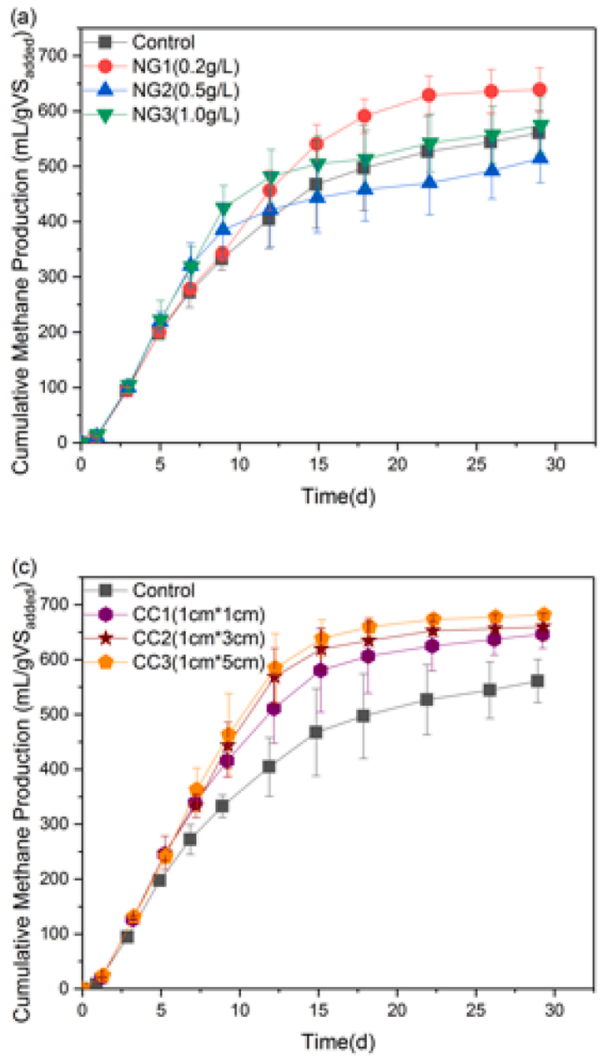

\section{Results and discussion}

\subsection{Influence of carbon-based CMs on co-digestion performance}

\subsubsection{Methane production}

The maximum amount of methane recovered from anaerobic codigestion of FOG and WAS was dependent on the types and dosages of carbon-based CMs (Fig. 1). For NG (Fig. 1a), the highest cumulative methane production was obtained with the lowest dosage at $0.2 \mathrm{~g} / \mathrm{L}$ (NG1), which is $14 \%$ higher than the cumulative methane production of Control. High conductivity of NG may induce DIET to enhance the methane production. Nevertheless, the cumulative methane production of NG2 was lower than that of the control, suggesting that the addition of NG could cause negative effect on methane production, which may be attributed to the antibacterial activity from the direct contact with sharp graphite surface disrupting cell membranes (Liu et al., 2011; Zhu et al., 2018). Unlike NG, the cumulative methane production of all tests with GAC addition was higher than that of Control (Fig. 1b). The highest cumulative methane production was obtained at the dosage of $10 \mathrm{~g} / \mathrm{L}$ (GAC2), followed by GAC3 and GAC1, suggesting higher dosage (greater than $10 \mathrm{~g} / \mathrm{L}$ ) of GAC would not continue to enhance methane production, which is consistent with the previous finding that GAC addition concentrations higher than $10 \mathrm{~g} / \mathrm{L}$ could hinder methanogenic activity (Rasapoor et al., 2020; Zhu et al., 2018). There are two possible explanations for this observation. The first concerns the adsorption of ammonia, which may harm the methanogens on the GAC surface (Rasapoor et al., 2020; Yin and Wu, 2019). The existence of ammonia is supported by the observation that there were $37-40 \mathrm{mg} / \mathrm{L}$ ammonia left after 30 days incubation for all GAC reactors (Table 2). The other possible explanation is the adsorption of LCFAs on GAC, which may inhibit methanogens and prevent biodegradation of LCFAs (Chowdhury et al., 2019; Sousa et al., 2009). The scenario of CC addition was different that, as the surface area increased from CC1 to CC3, the cumulative methane production was $15 \%$ to $22 \%$ higher than that of
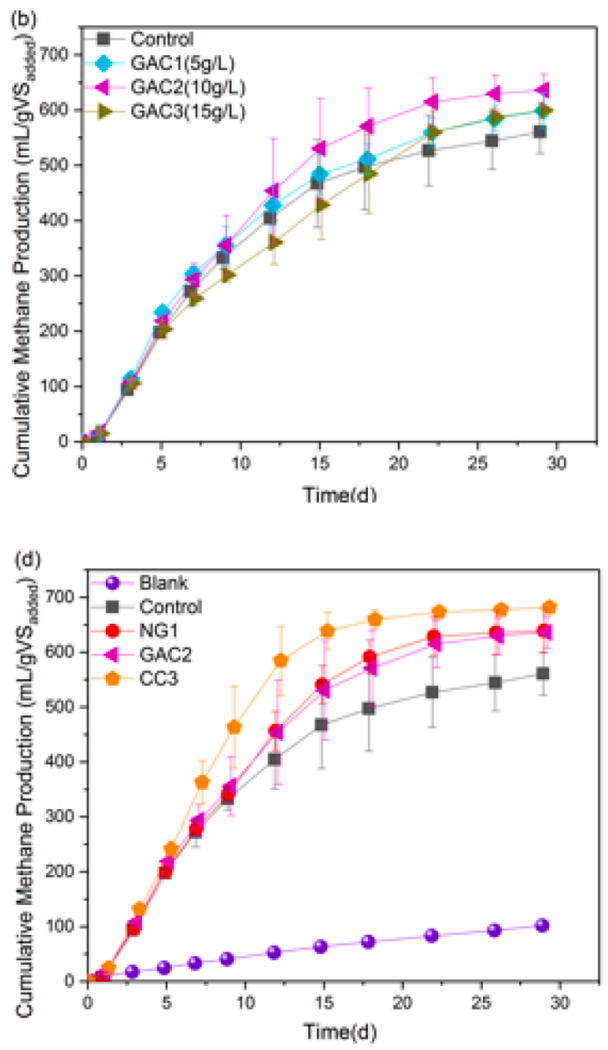

Fig. 1. Influence of different types and dosages of conductive materials on methane production during anaerobic digestion of FOG and WAS. 
Table 2

Ammonia-nitrogen concentrations and $\mathrm{pH}$ of all the reactors after 30 days incubation.

\begin{tabular}{lll}
\hline & Ammonia-nitrogen concentration $(\mathrm{mg} / \mathrm{L})$ & $\mathrm{pH}$ \\
\hline Control & $60.49 \pm 2.09$ & $7.69 \pm 0.11$ \\
NG1 & $38.50 \pm 8.56$ & $7.82 \pm 0.19$ \\
NG2 & $55.45 \pm 0.32$ & $7.59 \pm 0.23$ \\
NG3 & $56.64 \pm 0.18$ & $7.67 \pm 0.27$ \\
GAC1 & $39.56 \pm 5.12$ & $7.24 \pm 0.02$ \\
GAC2 & $37.71 \pm 2.65$ & $7.33 \pm 0.03$ \\
GAC2 & $39.47 \pm 1.47$ & $7.33 \pm 0.09$ \\
CC1 & $43.26 \pm 6.48$ & $7.56 \pm 0.11$ \\
CC2 & $40.58 \pm 4.14$ & $7.61 \pm 0.03$ \\
CC3 & $37.95 \pm 3.98$ & $7.55 \pm 0.18$ \\
\hline
\end{tabular}

Control (Fig. 1c). This may be due to the high conductivity and large surface of CC with no sharp contact with microorganisms and low adsorption of ammonia or LCFAs. Comparison was made among the best scenario of NG, GAC, and CC treatments, showing that CC3 had the highest cumulative methane production, followed by NG1 and GAC2 (Fig. 1d).

Two peaks of daily methane production rate were observed in treatments with the addition of CMs while only one peak was found in the control (Fig. 2), suggesting that the second peak was responsible for the higher methane production by the presence of CMs. The pattern of the first peak in NG1, GAC2, and Control were almost the same while the first peak of CC3 was slightly higher. The decline of the first peak was probably due to the inhibitory effect of LCFAs (product of FOG hydrolysis) on anaerobic microbes (Hao et al., 2020). Nevertheless, this inhibitory effect was little by the presence of CC as the second peak in CC3 was much earlier and higher than those in NG1 and GAC2, which may be due to the high capacity of CC to resist acid impacts (Martins et al., 2018; Zhao et al., 2017). A regression analysis of experimental data indicated that the cumulative methane production fit well with the modified Gompertz model with an $\mathrm{R}^{2}$ value of $0.98-0.99$ (Table 3 ). The regression analysis confirmed that CC3 had the highest cumulative methane production and maximum daily methane production rate $(R \mathrm{~m})$. Compared to the control, the lag phase $(\lambda)$ was barely changed in $C C$ and NG reactors but significantly reduced by the presence of GAC, while the $R \mathrm{~m}$ was enhanced in CC and NG reactors but not in GAC reactors. This suggested that all materials are unique though they may have similar physical or chemical behavior, and the way that they affect the biological reactions can be potentially different. Even for a specific CM with

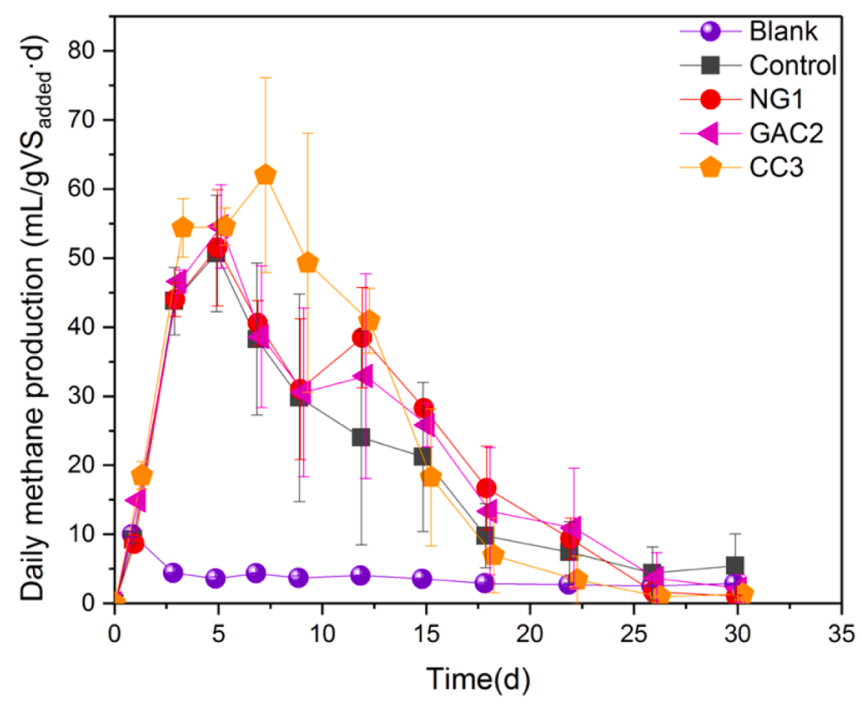

Fig. 2. Influence of different types and dosages of conductive materials on daily methane production during anaerobic digestion of FOG and WAS. different dosages, the highest cumulative methane production was not necessarily obtained from the reactor with relatively short $\lambda$ and high $R m$. Take NG for example, NG2 had lowest cumulative methane production but with relatively short $\lambda$ and high $R m$ while NG1 had the highest cumulative methane production but with $\mathrm{Rm}$ much lower than that of NG2 or NG3. This is due to the change pattern of daily methane production (see supplementary materials) that, though $R m$ was obtained from the first peak for all NG reactors, the second peak of NG1 was much wider and higher than that of NG2 and NG3 resulting in the highest cumulative methane production in NG1, which supports our hypothesis that the second peak of daily methane production was essential for the enhanced methane production by the presence of CMs.

\subsubsection{VS removal and COD conversion efficiency}

The VS removal was also influenced by the addition of CMs (Table 4). Compared to the control (21.4\% of VS removal), higher VS removal rates were observed for all treatments with CM addition except for NG2, suggesting a positive effect on FOG degradation and WAS disposal. The VS removal efficiency was dependent on the type and dosage of CMs, which is in line with the change pattern of cumulative methane production in all treatments with $\mathrm{CM}$ addition. For a specific type of CM, the highest VS removal rate was $55.6 \%, 46.1 \%$ and $76.3 \%$ in NG1, GAC2 and CC3, respectively. Similar scenario was found in COD conversion efficiency (determined by the ratio of measured ultimate methane production over theoretical maximum methane production (Nielfa et al., 2015)) (Table 5). Compared to the control (51.0\%), the COD conversion efficiency was increased to $58.7 \%, 58.5 \%$, and $62.7 \%$ in NG1, GAC2, and CC3, respectively. Taken together, these results show that the change patterns of cumulative methane production, VS removal, and COD conversion efficiency were consistent for a specific CM. Addition of $\mathrm{CM}$ did not necessarily result in enhanced performance during anaerobic co-digestion of FOG and WAS, which was dependent on the type and dosage of CMs.

\subsection{Characterization of aggregates on CMs}

According to scanning electron microscopy (SEM) of CMs before and after incubation (see supplementary material), differences of surface structure were observed among the three CMs that NG as nanoparticles had a rough and sharp surface, the surface of GAC was relatively smooth with pores, and the line structure of CC provided large surface area for the attachment of microorganisms. The SEM of NG-supplemented cultures revealed that microorganisms could attach on the surface of NG but the surface was not fully covered by microorganisms. In contrast, more microorganisms attached on the surface of GAC and they were in a closer physical contact than that of NG. Regarding the CC-supplemented culture, microorganisms were not just attached on the cloth lines but also formed aggregations among lines. These observations demonstrated that the characterization of the surface structure had impact on the attachment and aggregation of microorganisms, which may consequently affect the number of microorganisms in the reaction system. The result of qPCR analysis supports this hypothesis, showing that the total bacterial and archaeal gene concentrations were higher in NG1, GAC2, and CC3 than those in the control, which were gradually increased from $4.7 \times 10^{12}$ to $2.0 \times 10^{13}$ copies/gVS for bacteria) and from $2.4 \times 10^{14}$ to $9.9 \times 10^{14}$ copies/gVS for archaea, respectively (Fig. 3). Though the total amount of bacteria and archaea in NG1 was almost half of that in GAC2, the conductivity of NG was much higher than that of GAC (see supplementary material), which may be the explanation for the slightly higher cumulative methane production in NG1 than that in GAC2. Without antibacterial activity or ammonia/LCFA adsorption, the large surface and high conductivity of CC resulted in the highest accumulation bacteria and archaea working together to achieve the biggest enhancement of methane production in CC3. 
Table 3

Kinetic parameters estimate from the modified Gompertz model for digesiton experiment.

\begin{tabular}{|c|c|c|c|c|c|c|c|c|c|c|}
\hline Parameters & Control & NG1 & NG2 & NG3 & GAC1 & GAC2 & GAC3 & CC1 & CC2 & CC3 \\
\hline Measured B (mL/gVS) & 561.0 & 639.0 & 514.7 & 574.9 & 599.0 & 636.3 & 599.9 & 646.5 & 659.7 & 681.6 \\
\hline Estimated $\mathrm{B}_{0}(\mathrm{~mL} / \mathrm{gVS})$ & 570.8 & 648.5 & 478.8 & 548.2 & 578.9 & 636.8 & 613.6 & 638.9 & 662.6 & 683.4 \\
\hline$\lambda(\mathrm{d})$ & 1.5 & 1.3 & 1.3 & 1.4 & 0.5 & 1.0 & 0.2 & 1.2 & 1.5 & 1.6 \\
\hline $\mathrm{R}_{\mathrm{m}}(\mathrm{mL} / \mathrm{gVS} \cdot \mathrm{d})$ & 44.2 & 47.6 & 57.1 & 59.5 & 43.5 & 44.0 & 33.2 & 55.7 & 62.4 & 65.8 \\
\hline $\mathrm{R}^{2}$ & 0.99 & 0.99 & 0.99 & 0.99 & 0.98 & 0.99 & 0.98 & 0.99 & 0.99 & 0.99 \\
\hline
\end{tabular}

Table 4

Initial and final VS of anaerobic digestion of FOG and WAS.

\begin{tabular}{llllll}
\hline & \multicolumn{2}{l}{ Initial VS $(\mathrm{mg})$} & & Final VS (mg) & \multirow{2}{*}{ VS removal (\%) } \\
\cline { 2 - 3 } & Inoculum & WAS & FOG & & \\
\hline Blank & 527 & & & 414 & $21.4 \%$ \\
Control & 527 & 47 & 216 & 596 & $24.6 \%$ \\
NG1 & 527 & 47 & 216 & 351 & $55.6 \%$ \\
NG2 & 527 & 47 & 216 & 658 & $16.7 \%$ \\
NG3 & 527 & 47 & 216 & 555 & $29.7 \%$ \\
GAC1 & 527 & 47 & 216 & 469 & $40.6 \%$ \\
GAC2 & 527 & 47 & 216 & 426 & $46.1 \%$ \\
GAC3 & 527 & 47 & 216 & 459 & $41.9 \%$ \\
CC1 & 527 & 47 & 216 & 320 & $59.5 \%$ \\
CC2 & 527 & 47 & 216 & 272 & $65.6 \%$ \\
CC3 & 527 & 47 & 216 & 187 & $76.3 \%$ \\
\hline
\end{tabular}

Table 5

COD conversion efficiency determined by the radio of measured ultimate methane production over theoretical maximum methane production.

\begin{tabular}{lllll}
\hline & $\begin{array}{l}\text { Initial } \\
\text { COD } \\
(\mathrm{mg})\end{array}$ & $\begin{array}{l}\text { Theoretical } \\
\text { maximum methane } \\
\text { production at STP } \\
\left(\mathrm{CH}_{4 \max }\right)(\mathrm{mL})\end{array}$ & $\begin{array}{l}\text { Measured } \\
\text { ultimate methane } \\
\text { production at STP } \\
\left(\mathrm{CH}_{4 \mathrm{f}}\right)(\mathrm{mL})\end{array}$ & $\begin{array}{l}\text { Conversion } \\
\text { efficiency } \\
\left(\mathrm{CH}_{4 \mathrm{f}} / \mathrm{CH}_{4 \mathrm{max}}\right) \\
(\%)\end{array}$ \\
\hline Blank & 499 & 174.7 & 53.6 & $30.7 \%$ \\
Control & 817 & 286 & 147.5 & $51.0 \%$ \\
NG1 & 817 & 286 & 168.0 & $58.7 \%$ \\
NG2 & 817 & 286 & 135.4 & $47.3 \%$ \\
NG3 & 817 & 286 & 151.2 & $52.9 \%$ \\
GAC1 & 817 & 286 & 157.5 & $55.1 \%$ \\
GAC2 & 817 & 286 & 167.3 & $58.5 \%$ \\
GAC2 & 817 & 286 & 157.8 & $55.2 \%$ \\
CC1 & 817 & 286 & 170 & $59.4 \%$ \\
CC2 & 817 & 286 & 173.5 & $60.7 \%$ \\
CC3 & 817 & 286 & 179.3 & $62.7 \%$ \\
\hline
\end{tabular}

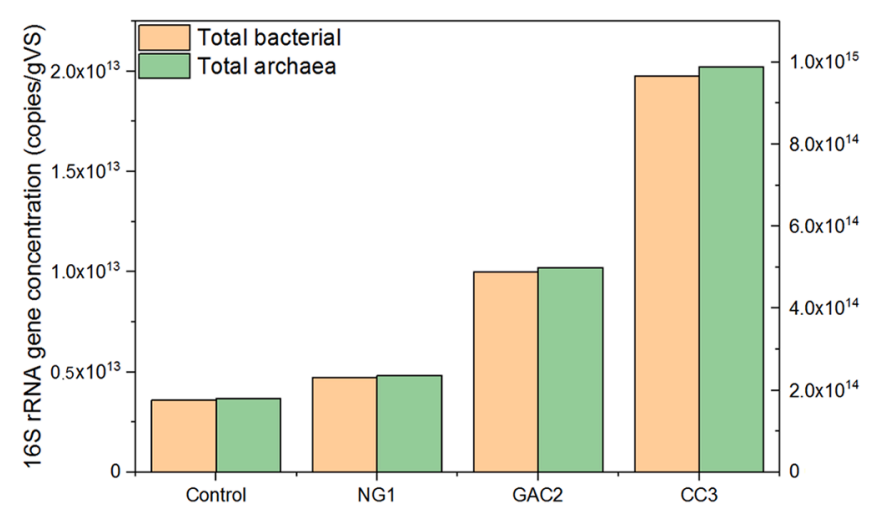

Fig. 3. qPCR result of total bacteria and archaea in Control, NG1, GAC2, and CC3.

\subsection{Microbial communities}

The interactions among key microorganisms involved in C bioconversions are essential for elucidating the mechanisms for the FOG degradation and biogas production. A total of 18 samples were sequenced, generating 58,213 quality-filtered and chimera-free sequences with a total of 2590 OTUs identified. Overall dissimilarity of bacterial and archaeal communities is shown in Fig. 4 using principal coordinate analysis (PCoA). Relatively high reproducibility in triplicate samples was obtained for all samples except for the attached samples on carbon cloth (CC3-A). Apart from the inoculum, bacterial communities were shifted into two potential clusters (Fig. 4a). Cluster 1 contained biomass samples from Control, NG1, and CC3-S (suspended sludge in CC3), suggesting a not significant dissimilarity of bacterial communities among them. Biomass samples in Cluster 2 (GAC2 and CC3-A) were not clustered closer than those in Cluster 1 due to the big variation among the triplicates of CC3-A. Separation of the two clusters indicates that the difference of bacterial community composition was developed based on the addition of different types of CMs. Only one cluster was found for archaeal communities (Fig. 4b), which contained the samples from Control and NG1, showing similar archaeal communities between Control and NG1. Dissimilarity was observed for the archaeal communities of Inoculum, GAC2, CC3-S, and CC3-A as they were scatteredly distributed, implying a greater impact of $\mathrm{CM}$ addition on the composition of archaeal communities than that of bacterial communities. The similarity of bacterial and archaeal communities between Control and NG1 suggested that small changes of community composition might be responsible for the enhanced methane production by the presence of NG.

Twenty-one of the most abundant OTUs were detected in bacterial communities (Fig. 5) while ten of the most abundant OTUs were detected in archaeal communities (Fig. 6), all with relative abundance $>1.5 \%$. Although similarity of microbial communities was found between NG1 and Control, differences of microbial community composition were observed that the relative abundances of OTUs assigned to genus Exilispira, class Thermococci, and genus Candidatus Methanomethylicus were higher in NG1 than those in Control. Exilispira belongs to the phylum Spirochaetes and strong evidence has been provided to indicate the involvement of Spirochaetes in syntrophic acetate oxidation (SAO) generating $\mathrm{H}_{2}$ and $\mathrm{CO}_{2}$ as metabolic products (Lee et al., 2015, 2013). Species affiliated with Thermococci are likely to ferment organic compounds and produce hydrogen to support the growth of hydrogenotrophic methanogens through syntrophy (Hensley et al., 2016; Price et al., 2015). Since the hydrogenotrophic methanogen Methanobacterium was dominant in the archaeal community of NG1, it is likely that the supplementation of NG enhanced the acetate conversion through SAO between Spirochaetes and Methanobacterium for higher methane production. Besides, Candidatus Methanomethylicus, which is inferred to be capable of methylotrophic methanogenesis (Vanwonterghem et al., 2016), was in higher abundance in NG1 than that in Control, suggesting that the addition of NG might enhance methylotrophic methanogenesis contributing to the methane production in NG1. Previous study has shown that Methanosaeta can make direct electrical connections with Geobacter to enhance methane production during anaerobic digestion (Rotaru et al., 2014). Though Geobacter and Methanosaeta were detected in NG1, the supplementation of NG did not significantly stimulate methane production through DIET between Geobacter and Methanosaeta as their abundances in NG1 were almost the same as those in Control.

Clear shift of microbial community was observed between Control and GAC2 that Geobacter and Methanosaeta were both in higher 

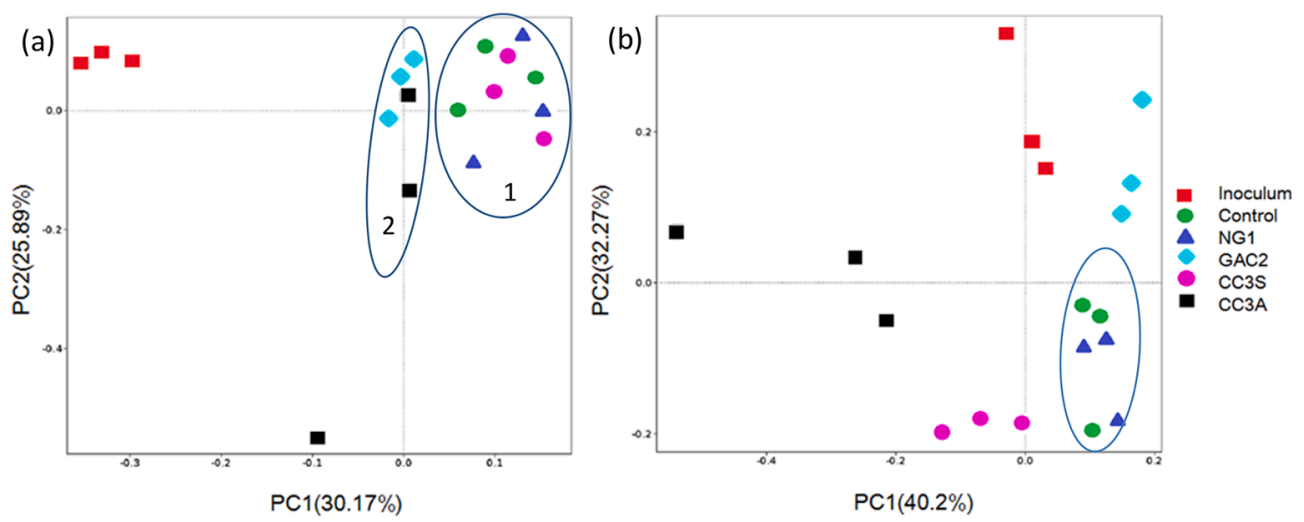

Fig. 4. Bacterial (a) and archaeal (b) community dissimilarity of inoculum and biomass samples from digestions applying principal coordinate analysis (PCoA) based on Bray-Curtis distance.
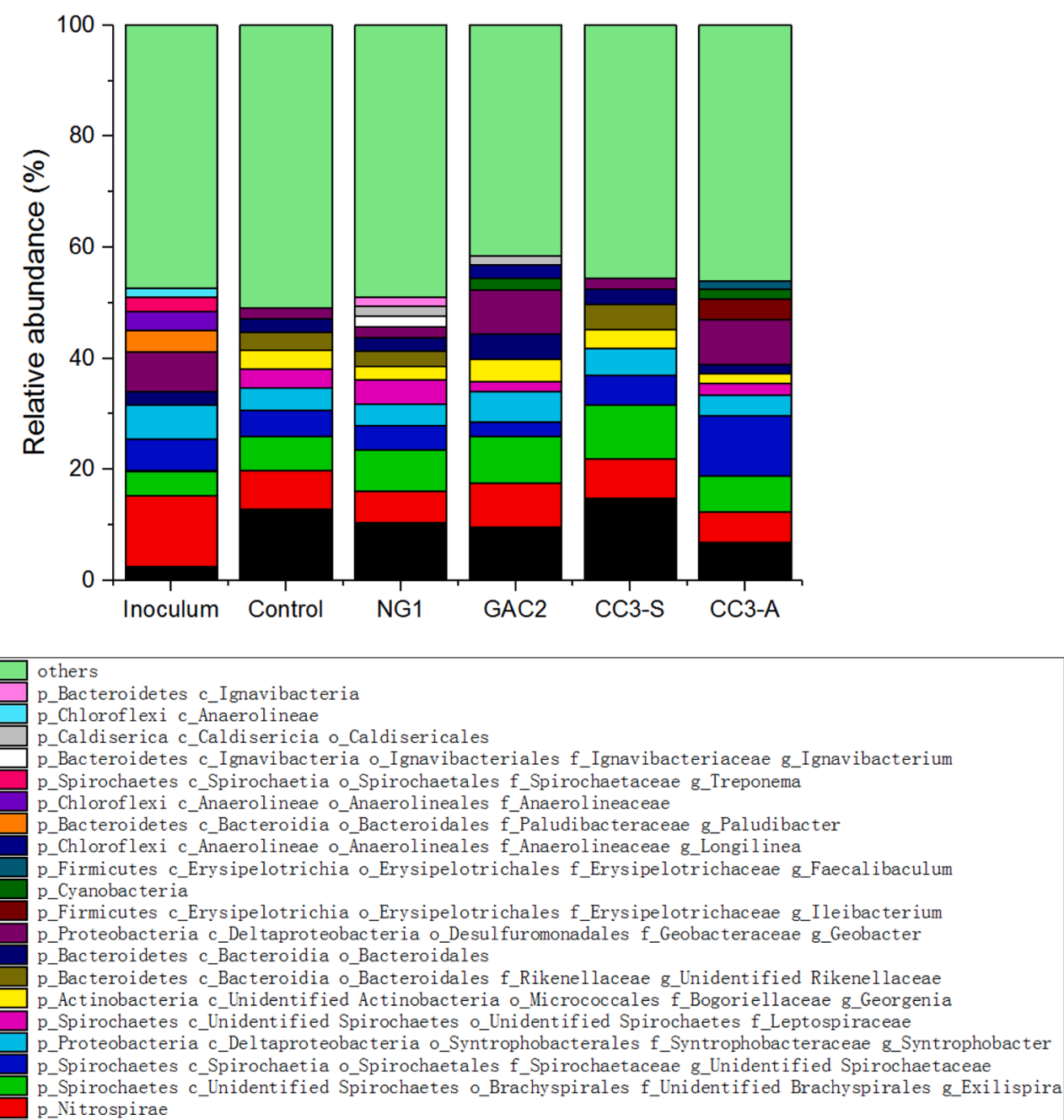

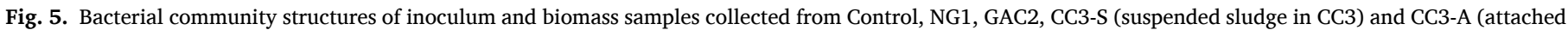
sludge on CC) at the end of experimental period.

abundances in GAC2, suggesting the occurrence of DIET between them to enhance the methane production in GAC2. Furthermore, Exilispira and Thermococci were greatly enriched in GAC2. Though the relative abundance of Methanobacterium was lower in GAC2 than that in Control, another hydrogenotrophic methanogen Methanolinea were highly enriched in GAC2. Due to the possible roles of Exilispira, Thermococci, and hydrogenotrophic methanogens involved in SAO (as discussed above), the presence of GAC may also facilitate SAO during anaerobic co-digestion of FOG and WAS. However, substantial enrichment of Candidatus Methanomethylicus was not obtained in GAC2, showing that methylotrophic methanogenesis might not be responsible for the enhanced methane production in GAC2. 


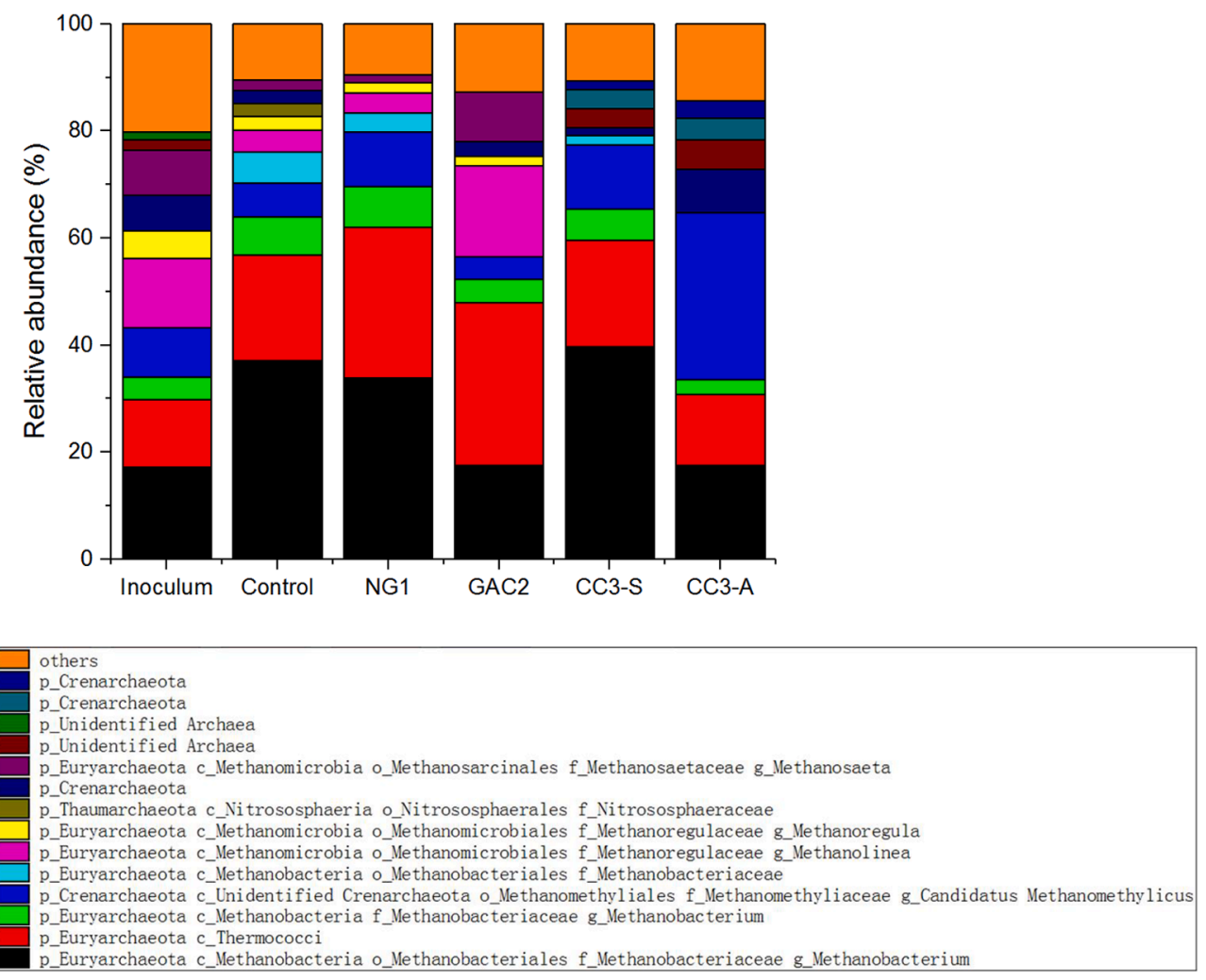

Fig. 6. Archaeal community structures of inoculum and biomass samples collected from Control, NG1, GAC2, CC3-S (suspended sludge in CC3) and CC3-A (attached sludge on $\mathrm{CC}$ ) at the end of experimental period.

With the presence of CC, Spirochaetaceae, Geobacter, and Candidatus Methanomethylicu were enriched in higher abundances in CC3-A than in CC3-S and Control, while Ileibacterium and Faecalibaculum belonging to phylum Firmicutes were enriched in CC3-A but rarely found in other samples (even in CC3-S), showing the possible involvement of these species in DIET as well as the difference of community composition between CC3-A and CC3-S/samples in other rectors. Similar as the scenario in NG1 and GAC2, Exilispira was dominant in CC3 but with a higher relative abundance in the suspended sludge than in the attached biomass on the surface of CC, and the enrichment of Spirochaetaceae and Exilispira (both in phylum Spirochaetes) were likely to accelerate the acetate conversion through SAO with the assistance of Methanobacterium (dominant archaea in CC3). However, the relative abundance of Methanosaeta was quite low in CC3 despite Geobacter was present in a relatively high abundance in the suspended sludge and greatly enriched on the surface of CC, suggesting that DIET between Methanosaeta and Geobacter was rarely occurred in CC3. Instead, the OTUs assigned to phylum Crenarchaeota (e.g., Candidatus Methanomethylicu) were highly enriched. Previous studies have only been performed to investigate the electrical connection between Geobacter and hydrogenotrophic methanogens (e.g., Methanospirillum (Walker et al., 2019)) or acetoclastic methanogens (such as Methanosaeta and Methanosarcina (Rotaru et al., 2014, 2015)), but not for methylotrophic methanogens yet. Further study is needed to investigate the diversity of methanogens capable of DIET.

Though acetoclastic methanogenesis was found to be the major pathway in many studies for anaerobic digestion of FOG (Chowdhury et al., 2019; Kurade et al., 2019; Nakasaki et al., 2020), acetoclastic methanogens were not dominant in this study. Since acetoclastic methanogens are considered to be most sensitive to ammonia (Rajagopal et al., 2013), high ammonia could result in the appearance of microbial competitors for acetate conversion and promote the development of SAO, which has been observed in many ammonia-stressed systems during anaerobic digestion (Westerholm et al., 2016). The possible inhibitory effect of ammonia on acetoclastic methanogens is supported by three observations in this study: 1) the OTU assigned to Nitrospirae (species in Nitrospirae are very likely to participate in reactions with nitrogenous compounds) was dominant in all reactors, suggesting the presence of a relatively high concentration of nitrogenous compounds ; 2) the ammonia-nitrogen concentration in all reactors were not low at the end of 30 days incubation (Table 2); 3 ) the $\mathrm{pH}$ of all reactors were ranged from 7.22 to 8.00 (Table 2), which could promote the shift of total ammonia-nitrogen to free ammonia that is reported to be the main cause of inhibition due to its high permeability to cell membrane leading to proton imbalance and potassium deficiency (especially for acetoclastic methanogens) (Rajagopal et al., 2013). Moreover, the pathway of acetate conversion shifted to SAO is also supported by our observation of the dominance of Spirochaetes and hydrogenotrophic methanogens in all reactors. Meanwhile, the enrichment of Methanosaeta (acetoclastic methanogen) in GAC2 may be due to the adsorption of ammonia on GAC that alleviated the inhibitory effect of ammonia on methanogens in the reactor as well as the high affinity of Methanosaeta for acetate when acetate concentration (below $1 \mathrm{mM}$, data not shown) was low (Smith and Ingram-Smith, 2007).

Addition of CMs resulted in the further promotion of SAO in NG1, GAC2 and CC3 (as previous data showed), which could help to accelerate the degradation of LCFAs as acetate and hydrogen are the productions of LCFA degradation through $\beta$-oxidation (Sousa et al., 2009). One possible reason is the aggregation of microbes on the surface of CMs to support SAO bacteria (despite their slow growth rate) and reduce distance between SAO bacteria and hydrogenotrophic methanogens that could facilitate the interspecies hydrogen transfer. Another possible explanation concerns the DIET-mediated SAO, allowing much more quick acetate conversion as the electron transfer speed with DIET is $10^{6}$ times faster than indirect interspecies transfer (Cruz Viggi et al., 2014; Zhang et al., 2018). Nevertheless, the functional microbes involved in DIET-mediated SAO were different and dependent on the types of CMs as Geobacter, which specialize in making electrical contacts with 
extracellular electron acceptors or other organisms, were highly enriched in GAC2 and CC3-A but not in NG1. Although the syntrophic DIET function has not been demonstrated with Methanobacterium, some studies found recently that Geobacter and Methanobacterium were potentially involved in DIET-mediated SAO for enhanced methane production in anaerobic digesters (Shen et al., 2020; Zhuang et al., 2018). It is worth noting that the cumulative methane production in NG1 was slightly higher than that in GAC2 despite the relative abundance of Geobacter and the total amounts of bacteria and archaea were significantly lower in NG1. This implies that, apart from Geobacter, other species belonging to Exilispira, Thermococci, and Candidatus Methanomethylicus might be capable of DIET to enhance methane production, which deserves further investigation.

\section{Conclusion}

Carbon-based CMs were evaluated when they were added to the anaerobic co-digestion of FOG and WAS. The best scenario was achieved at CC3 with the most incremental methane production (22\%). The impact of CM addition on the composition of archaeal communities was greater than that of bacterial communities. The DIET-mediated SAO enabling faster acetate conversion might be responsible for the promotion of SAO by the presence of CMs, leading to the enhancement of methane production. These findings suggested that application of carbon-based CMs appears as a good strategy to accelerate methane production rate during anaerobic digestion of FOG.

\section{CRediT authorship contribution statement}

Xia He: Conceptualization, Formal analysis, Investigation, Methodology, Data curation, Funding acquisition, Resources, Writing - original draft. Zhenyu Guo: Formal analysis, Investigation, Methodology. Jian Lu: Conceptualization, Funding acquisition, Methodology, Project administration, Supervision, Resources, , Writing - review \& editing. Ping Zhang: Methodology, Writing - review \& editing.

\section{Declaration of Competing Interest}

The authors declare that they have no known competing financial interests or personal relationships that could have appeared to influence the work reported in this paper.

\section{Acknowledgments}

This work was financially supported by the National Natural Science Foundation of China (41877131), One Hundred Talents Program of Guangxi Autonomous Region (No. 2017-53), Taishan Scholars Program of Shandong Province (No. tsqn201812116), Science and Technology Service Network Initiative of the Chinese Academy of Sciences (KFJSTS-QYZX-114), and Two-Hundred Talents Plan of Yantai (Y739011021).

\section{Appendix A. Supplementary data}

Supplementary data to this article can be found online at https://doi. org/10.1016/j.biortech.2021.124871.

\section{References}

APHA, 1999. Standard Methods for Examination of Water and Wastewater., American Public Health Association, American Water Works Association and Water Environmental Federation: Washington, DC, USA.

Chen, S., Rotaru, A.E., Liu, F., Philips, J., Woodard, T.L., Nevin, K.P., Lovley, D.R., 2014 Carbon cloth stimulates direct interspecies electron transfer in syntrophic cocultures. Bioresour. Technol. 173, 82-86.

Chowdhury, B., Lin, L., Dhar, B.R., Islam, M.N., McCartney, D., Kumar, A., 2019. Enhanced biomethane recovery from fat, oil, and grease through co-digestion with food waste and addition of conductive materials. Chemosphere 236, 124362.
Cruz Viggi, C., Rossetti, S., Fazi, S., Paiano, P., Majone, M., Aulenta, F., 2014. Magnetite particles triggering a faster and more robust syntrophic pathway of methanogenic propionate degradation. Environ. Sci. Technol. 48, 7536-7543.

Fan, X., Xing, P., 2016. Differences in the composition of archaeal communities in sediments from contrasting zones of Lake Taihu. Front. Microbiol. 7, 1-11.

Hao, J., de los Reyes, F.L., He, X., 2020. Fat, oil, and grease (FOG) deposits yield higher methane than FOG in anaerobic co-digestion with waste activated sludge. J. Environ. Manage. 268, 110708.

Harris, P.W., Mccabe, B.K., 2015. Review of pre-treatments used in anaerobic digestion and their potential application in high-fat cattle slaughterhouse wastewater. Appl. Energy 155, 560-575.

He, X., de los Reyes, F.L., Ducoste, J.J., 2017. A critical review of fat, oil, and grease (FOG) in sewer collection systems: challenges and control. Crit. Rev. Environ. Sci. Technol. 47, 1191-1217.

Hensley, S.A., Moreira, E., Holden, J.F., 2016. Hydrogen production and enzyme activities in the hyperthermophile Thermococcus paralvinellae grown on maltose, tryptone, and agricultural waste. Front. Microbiol. 7, 1-13.

Kurade, M.B., Saha, S., Salama, E.S., Patil, S.M., Govindwar, S.P., Jeon, B.H., 2019. Acetoclastic methanogenesis led by Methanosarcina in anaerobic co-digestion of fats, oil and grease for enhanced production of methane. Bioresour. Technol. 272, 351-359.

Lee, S.H., Park, J.H., Kang, H.J., Lee, Y.H., Lee, T.J., Park, H.D., 2013. Distribution and abundance of Spirochaetes in full-scale anaerobic digesters. Bioresour. Technol. 145, 25-32.

Lee, S.H., Park, J.H., Kim, S.H., Yu, B.J., Yoon, J.J., Park, H.D., 2015. Evidence of syntrophic acetate oxidation by Spirochaetes during anaerobic methane production. Bioresour. Technol. 190, 543-549.

Li, C., Champagne, P., Anderson, B.C., 2011. Evaluating and modeling biogas production from municipal fat, oil, and grease and synthetic kitchen waste in anaerobic codigestions. Bioresour. Technol. 102, 9471-9480.

Liu, S., Zeng, T.H., Hofmann, M., Burcombe, E., Wei, J., Jiang, R., Kong, J., Chen, Y., 2011. Antibacterial activity of graphite, graphite oxide, graphene oxide, and reduced graphene oxide: Membrane and oxidative stress. ACS Nano 5, 6971-6980.

Long, J.H., Aziz, T.N., Reyes, F.L.D.L., Ducoste, J.J., 2012. Anaerobic co-digestion of fat, oil, and grease (FOG): a review of gas production and process limitations. Process Saf. Environ. Prot. 90, 231-245.

Lovley, D.R., 2017. Syntrophy goes electric: direct interspecies electron transfer. Annu. Rev. Microbiol. 71, 643-664.

Lu, J., Lin, Y., Wu, J., Zhang, C., 2021. Continental-scale spatial distribution, sources, and health risks of heavy metals in seafood: challenge for the water-food-energy nexus sustainability in coastal regions? Environ. Sci. Pollut. Res. in press.

Lü, F., Liu, Y., Shao, L., He, P., 2019. Powdered biochar doubled microbial growth in anaerobic digestion of oil. Appl. Energy 247, 605-614.

Martins, G., Salvador, A.F., Pereira, L., Alves, M.M., 2018. Methane production and conductive materials: a critical review. Environ. Sci. Technol. 52, 10241-10253.

Mata-alvarez, J., Dosta, J., Romero-güiza, M.S., Fonoll, X., Peces, M., Astals, S., 2014. A critical review on anaerobic co-digestion achievements between 2010 and 2013. Renew. Sustain. Energy Rev. 36, 412-427.

Mu, L., Zhang, L., Zhu, K., Ma, J., Li, A., 2018. Semi-continuous anaerobic digestion of extruded OFMSW: Process performance and energetics evaluation. Bioresour. Technol. 247, 103-115.

Nakasaki, K., Nguyen, K.K., Ballesteros, F.C., Maekawa, T., Koyama, M., 2020. Characterizing the microbial community involved in anaerobic digestion of lipidrich wastewater to produce methane gas. Anaerobe 61, 102082.

Navas-Molina, J.A., Peralta-Sánchez, J.M., González, A., McMurdie, P.J., VázquezBaeza, Y., Xu, Z., Ursell, L.K., Lauber, C., Zhou, H., Song, S.J., Huntley, J., Ackermann, G.L., Berg-Lyons, D., Holmes, S., Caporaso, J.G., Knight, R., 2013. Advancing our understanding of the human microbiome using QIIME. Methods Enzymol.

Nielfa, A., Cano, R., Fdz-Polanco, M., 2015. Theoretical methane production generated by the co-digestion of organic fraction municipal solid waste and biological sludge. Biotechnol. Rep. 5, 14-21.

Palatsi, J., Laureni, M., Andrés, M.V., Flotats, X., Nielsen, H.B., Angelidaki, I., 2009. Strategies for recovering inhibition caused by long chain fatty acids on anaerobic thermophilic biogas reactors. Bioresour. Technol. 100, 4588-4596.

Price, M.T., Fullerton, H., Moyer, C.L., 2015. Biogeography and evolution of Thermococcus isolates from hydrothermal vent systems of the Pacific. Front. Microbiol. 6, 1-12.

Rajagopal, R., Massé, D.I., Singh, G., 2013. A critical review on inhibition of anaerobic digestion process by excess ammonia. Bioresour. Technol. 143, 632-641.

Rasapoor, M., Young, B., Asadov, A., Brar, R., Sarmah, A.K., Zhuang, W.Q., Baroutian, S., 2020. Effects of biochar and activated carbon on biogas generation: a

thermogravimetric and chemical analysis approach. Energy Convers. Manage. 203, $1-10$.

Rotaru, A.E., Shrestha, P.M., Liu, F., Shrestha, M., Shrestha, D., Embree, M., Zengler, K., Wardman, C., Nevin, K.P., Lovley, D.R., 2014. A new model for electron flow during anaerobic digestion: Direct interspecies electron transfer to Methanosaeta for the reduction of carbon dioxide to methane. Energy Environ. Sci. 7, 408-415.

Rotaru, A.E., Woodard, T.L., Nevin, K.P., Lovley, D.R., 2015. Link between capacity for current production and syntrophic growth in Geobacter species. Front. Microbiol. 6, $1-8$.

Salama, E.-S., Saha, S., Kurade, M.B., Dev, S., Chang, S.W., Jeon, B.-H., 2019. Recent trends in anaerobic co-digestion: fat, oil, and grease (FOG) for enhanced biomethanation. Prog. Energy Combust. Sci. 70, 22-42. 
Shen, N., Liang, Z., Chen, Y., Song, H., Wan, J., 2020. Enhancement of syntrophic acetate oxidation pathway via single walled carbon nanotubes addition under high acetate concentration and thermophilic condition. Bioresour. Technol. 306, 123182.

Silva, S.A., Salvador, A.F., Cavaleiro, A.J., Pereira, M.A., Stams, A.J.M., Alves, M.M., Sousa, D.Z., 2016. Toxicity of long chain fatty acids towards acetate conversion by Methanosaeta concilii and Methanosarcina mazei. Microb. Biotechnol. 9, 514-518.

Smith, K.S., Ingram-Smith, C., 2007. Methanosaeta, the forgotten methanogen? Trends Microbiol. 15, 150-155.

Sousa, D.Z., Smidt, H., Alves, M.M., Stams, A.J.M., 2009. Ecophysiology of syntrophic communities that degrade saturated and unsaturated long-chain fatty acids. FEMS Microbiol. Ecol. 68, 257-272.

Sundberg, C., Al-Soud, W.A., Larsson, M., Alm, E., Yekta, S.S., Svensson, B.H., Sørensen, S.J., Karlsson, A., 2013. 454 pyrosequencing analyses of bacterial and archaeal richness in 21 full-scale biogas digesters. FEMS Microbiol. Ecol. 85, 612-626.

Vanwonterghem, I., Evans, P.N., Parks, D.H., Jensen, P.D., Woodcroft, B.J., Hugenholtz, P., Tyson, G.W., 2016. Methylotrophic methanogenesis discovered in the archaeal phylum Verstraetearchaeota. Nat. Microbiol. 1, 1-9.

Walker, D.J.F., Martz, E., Holmes, D.E., Zhou, Z., Nonnenmann, S.S., Lovley, D.R., 2019 The archaellum of methanospirillum hungatei is electrically conductive. MBio 10, $1-6$.

Westerholm, M., Moestedt, J., Schnürer, A., 2016. Biogas production through syntrophic acetate oxidation and deliberate operating strategies for improved digester performance. Appl. Energy 179, 124-135.

Wirth, R., Kovács, E., Maróti, G., Bagi, Z., Rákhely, G., Kovács, K.L., 2012. Characterization of a biogas-producing microbial community by short-read next generation DNA sequencing. Biotechnol. Biofuels 5, 41.
Wu, L.J., Kobayashi, T., Kuramochi, H., Li, Y.Y., Xu, K.Q., Lv, Y., 2018. High loading anaerobic co-digestion of food waste and grease trap waste: determination of the limit and lipid/long chain fatty acid conversion. Chem. Eng. J. 338, 422-431.

Wu, Y., Wang, S., Liang, D., Li, N., 2020. Conductive materials in anaerobic digestion: From mechanism to application. Bioresour. Technol. 298.

Yin, Q., Wu, G., 2019. Advances in direct interspecies electron transfer and conductive materials: Electron flux, organic degradation and microbial interaction. Biotechnol. Adv. 37, 107443.

Zhang, C., Lu, J., Wu, J., 2020a. One-step green preparation of magnetic seaweed biochar/sulfidated $\mathrm{Fe} 0$ composite with strengthen adsorptive removal of tetrabromobisphenol A through in situ reduction. Bioresour. Technol. 307, 123170.

Zhang, J., Zhang, R., Wang, H., Yang, K., 2020b. Direct interspecies electron transfer stimulated by granular activated carbon enhances anaerobic methanation efficiency from typical kitchen waste lipid-rapeseed oil. Sci. Total Environ. 704, 135282.

Zhang, J., Zhao, W., Zhang, H., Wang, Z., Fan, C., Zang, L., 2018. Recent achievements in enhancing anaerobic digestion with carbon- based functional materials. Bioresour. Technol. 266, 555-567.

Zhao, Z., Zhang, Y., Li, Y., Dang, Y., Zhu, T., Quan, X., 2017. Potentially shifting from interspecies hydrogen transfer to direct interspecies electron transfer for syntrophic metabolism to resist acidic impact with conductive carbon cloth. Chem. Eng. J. 313, $10-18$.

Zhu, H., Han, Y., Ma, Wencheng, Han, H., Ma, Weiwei, Xu, C., 2018. New insights into enhanced anaerobic degradation of coal gasification wastewater (CGW) with the assistance of graphene. Bioresour. Technol. 262, 302-309.

Zhuang, L., Ma, J., Yu, Z., Wang, Y., Tang, J., 2018. Magnetite accelerates syntrophic acetate oxidation in methanogenic systems with high ammonia concentrations. Microb. Biotechnol. 11, 710-720. 